\title{
Usefulness of gamma-glutamyl transpeptidase and mean corpuscular volume in alcoholic liver disease
}

\author{
Hemantha Kumara D. S. ${ }^{1}$, Muralidhara Krishna C.S..$^{2, *}$, H.L. Vishwanath ${ }^{3}$ \\ ${ }^{1}$ Tutor, ${ }^{2}$ Assistant Professor, ${ }^{3}$ Professor and HOD, Dept. of Biochemistry, ${ }^{1}$ Shimoga Institute of Medical Sciences, Shimoga, \\ Karnataka, ${ }^{2,3}$ Bangalore Medical College and Research Institute, Bangalore, Karnataka, India \\ *Corresponding Author: Muralidhara Krishna C.S. \\ Email: muralidharakrishna@yahoo.com
}

Received: $16^{\text {th }}$ February, 2018

Accepted: $24^{\text {th }}$ August, 2018

\begin{abstract}
Introduction: Excessive consumption of alcohol and alcoholism are widely observed risk factors for damage to health and social problems. Early identification of alcoholic liver disease could improve the possibility of early treatment and reduce damage to health and health costs. Our present study was anticipated to establish the association between the liver enzyme gamma-glutamyl transpeptidase and an haematocrit parameter mean corpuscular volume in alcohol liver disease patients.

Materials and Methods: Our study includes 100 subjects, with 50 cases and 50 controls. Serum was collected for the analyses of GGT, liver enzymes ALP, AST, ALT, total protein, albumin, bilirubin. Haemoglobin, Mean corpuscular volume, PCV and RBC were also analyzed. The statistical relationship between AST and ALT has been calculated. Statistical data analysis was performed by SPSS 20 software.

Results: The values of the GGT and MCV levels were significantly increased in patients compared to controls (p <0.001). The MCV level showed a significant statistical association with alcoholic liver disease patients. The degree of elevation of AST was greater than ALT in ALD.

Conclusion: The Overall verdict of this study shows that the increase in $\gamma$ GT and MCV levels in ALD patients can be used for added diagnostic markers for the diagnosis of alcoholic liver disease.
\end{abstract}

Keywords: Gamma glutamyl transpeptidase (GGT), Mean corpuscular volume (MCV), Alcoholic liver disease (ALD).

\section{Introduction}

Alcohol has been used in society for centuries and around the world for its properties to improve mood and taste. ${ }^{1}$ It is known that regular excessive consumption of alcohol causes a wide range of diseases and disorders. Consumption of alcohol increases promptly in developing regions of the world and is a major concern for health, showing a greater prevalence of use and associated problems. The organ liver is one of the most affected organs and with physiological disorders following the ingestion of alcohol. ${ }^{2}$ Alcoholic liver disease is a term that includes hepatic parenchymal manifestations of excessive alcohol consumption including alcoholic fatty liver, alcoholic hepatitis and hepatic fibrosis leading to liver cirrhosis. ${ }^{3}$

Alcohol is a common cause of liver cirrhosis in the world, including India and alcoholic liver disease is among the top five-six most common causes of death in the world. Consumption of alcohol has steadily increased in India over the past decade due to the increase in socioeconomic status. Chronic and excessive alcohol ingestion is a major cause of liver disease. ${ }^{4}$ Liver cirrhosis is usually caused by alcoholism and hepatitis $\mathrm{B}$ or $\mathrm{C}$, but has many other possible causes. The epidemiology of liver cirrhosis varies according to gender, ethnic groups and geographic distribution. ${ }^{5}$ Moderate alcohol consumption can lead to a wide range of medical, psychiatric and social problems. However, alcohol is also widely available and enjoyable, so attempts have been made to identify the levels of alcohol consumption in which alcoholrelated harm occurs.

The amount and duration of alcohol consumption also counts for the development of the disease. The overall threshold for the development of classic alcoholic liver disease in men is an intake of > 60-80 g / day of alcohol for 10 years. Ingestion of $>160 \mathrm{~g} /$ day is associated with a 25-30 fold increased risk of developing alcoholic cirrhosis.

Gender differences are the result of the unknown effects of estrogen and alcohol metabolism. It has also been hypothesized that social, immunological and hereditary factors play a major role in the progression of the pathogenic process. The study done by Matoff $\mathrm{O}$ et al, explains the histopathological changes; that has been occurred by consumption of alcohol for longer duration: those three manifestations are A) alcoholic fatty liver, B) Alcoholic hepatitis and C) Alcoholic cirrhosis. The accumulation of fat in the liver leads to fatty liver stage, and this stage is reversible by complete cessation of drinking of alcohol. The chronic alcoholism leads to inflammation of liver called alcoholic hepatitis. Finally further alcoholism leads to fibrosis stage called alcoholic cirrhosis. The metabolic products of alcohol, mainly acetaldehyde formed by the oxidation of ethanol excites the production of collagen. It is an irreversible phase of hepatocyte damage and is of micronodular type. ${ }^{6}$ Alcoholic liver disease raises the liver enzymes aspartate and alanine transaminases. More than $85 \%$ of alcoholic liver disease patients have 
an AST: ALT ratio of 2 or more. ${ }^{7}$ Early identification of excessive consumption of alcohol and alcoholism could improve the possibility of early treatment and reduce damage to health and health costs. ${ }^{8}$ An extensive variety of biochemical and haematological parameters was affected by regular excessive alcohol consumption. ${ }^{9,10}$

\section{Materials and Methods}

We included 100 subjects, with 50 cases and 50 controls in this study. The study was conducted at BMC \& RI, connected to Victoria Hospital, Bengaluru. Controls were selected from healthy patients. The clinically diagnosed and ultrasound suggestive findings suggestive of alcoholic liver disease cases were included in the study. Individuals with hypertension, diabetes mellitus, pancreatitis, renal failure, autoimmune diseases, haemolytic anaemia, or liver infections were excluded from the study.

Method of Analysis: Institutional ethical committee clearance was taken. After obtaining informed consent in writing, $5 \mathrm{ml}$ of venous blood was obtained by venepuncture under aseptic conditions, the samples were centrifuged and the separated serum was used for the estimation of total proteins, albumin, Bilirubin (total and direct), liver enzymes AST, ALT ALP and GGT. Haemoglobin, Mean corpuscular volume, PCV and RBC were analysed by an automated cell Coulter Counter analyser. The statistical association between AST and ALT was calculated.

The results were tabulated. Results on continuous measurements are presented on Mean \pm SD. The Statistical analysis of data was done by using software namely SAS 9.9, SPSS 19.0, Stata 10.4.

The results of cases and controls were compared by student' $t$ ' test. A ' $p$ ' value of $<0.05$ was considered significant. A ' $p$ ' value of $<0.0001$ was considered as highly significant.

\section{Results}

Table 1: Biochemical parameters among ALD and control groups

\begin{tabular}{|l|c|c|c|}
\hline \multicolumn{1}{|c|}{ Lab variables } & Controls & Cases & p value \\
\hline Total bilirubin(mg/dl) & $0.49 \pm 0.26$ & $8.37 \pm 7.97$ & $<0.0001$ \\
\hline Direct bilirubin(mg/dl) & $0.02 \pm 0.04$ & $3.83 \pm 3.79$ & $<0.0001$ \\
\hline Total protein(g/dl) & $7.48 \pm 0.47$ & $6.43 \pm 1.19$ & $<0.0001$ \\
\hline Albumin(g/dl) & $4.25 \pm 0.34$ & $2.07 \pm 0.66$ & $<0.0001$ \\
\hline Alkaline phosphatase (IU/L) & $87.86 \pm 19.29$ & $166.16 \pm 73.53$ & $<0.0001$ \\
\hline AST (IU/L) & $29.3 \pm 7.57$ & $127.23 \pm 97.01$ & $<0.0001$ \\
\hline ALT (IU/L) & $23.63 \pm 8.82$ & $41.33 \pm 36.49$ & $0.0112^{*}$ \\
\hline Gamma-GT (IU/L) & $22.56 \pm 9.06$ & $90.7 \pm 48.61$ & $<0.0001$ \\
\hline AST:ALT & $1.23 \pm 0.77$ & $3.07 \pm 2.65$ & $<0.0001$ \\
\hline
\end{tabular}

Table 2: Hematological parameters

\begin{tabular}{|l|c|c|c|}
\hline \multicolumn{1}{|c|}{ Lab variables } & Controls & Cases & p value \\
\hline $\mathrm{RBC}(\mathrm{million} / \mathrm{mm} 3)$ & $3.49 \pm 0.45$ & $2.92 \pm 0.19$ & $<0.0001$ \\
\hline $\mathrm{Hb}(\mathrm{g} / \mathrm{dl})$ & $13.48 \pm 1.13$ & $12.98 \pm 1.01$ & $>0.05$ \\
\hline $\mathrm{PCV}(\%)$ & $31.41 \pm 1.09$ & $39.23 \pm 3.76$ & $<0.0001$ \\
\hline $\mathrm{MCV}(\mathrm{fl} / \mathrm{l})$ & $97.90 \pm 6.77$ & $130 \pm 14.77$ & $<0.0001$ \\
\hline
\end{tabular}

\section{Discussion}

In Table 1, our study shows that the levels of alkaline phosphatase, AST and $\gamma$-GT were significantly higher $(\mathrm{p}<0.0001)$ in patients compared to controls without ALD. Total protein and albumin decreased significantly ( $\mathrm{p}<0.0001)$ in patients. The relationship between the frequency of cough and ALT was significantly higher $(\mathrm{p}<0.0001)$ in the patient group compared to control.

In Table 2, our study reveals that the levels of MCV and PCV were also significantly higher ( $p$ $<0.0001)$ in patients. Patients had significantly lower levels of RBC ( $p<0.0001)$. Hemoglobin levels in patients and control groups did not differ significantly ( $>0.05)$.
Our study shows that GGT in cases was significantly higher than in controls. Quite a lot of studies showed that there is significant elevation of GGT in ALD. ${ }^{11,12}$ GGT is one of the liver enzyme which helps in identifying the hepatobiliary disease, being elevated in most subjects with liver disease regardless of cause but more specifically in biliary tract diseases. GGT is such a liver enzyme which is elevated in most of the liver diseases like biliary obstructions, infectious hepatitis, liver neoplasms and drug intoxications. Elevated GGT levels were found in alcoholic hepatitis and also in heavy alcohol drinkers. ${ }^{13}$

Clerment and Chalmers et al showed that the association between SGOT and SGPT could be used to limit the cause of liver diseases. ${ }^{14}$ Typically, the AST / ALT ratio is about $1.5-2$ to 1 in alcoholic liver 
disease..$^{15}$ Our study showed that the ratio of SGOT to SGPT is 3, along with increases in both SGOT and SGPT levels. ${ }^{1,6,17}$ Like GGT, aminotransferases act not only as indicators of alcohol consumption, but also as indicators of hepatic alcohol damage. ${ }^{17}$

The present study showed significantly elevated MCV levels compared to controls.

A study by Pasqualetti et al showed that Mean Corpuscular Volume is one of the notable marker of alcoholism in chronic liver disease. ${ }^{18}$

The mean corpuscular volume has been recognized for many years as increasing with excessive alcohol consumption. In excessive consumption of alcohol, macrocytosis tends to occur in the presence of normal folate levels in majority cases. ${ }^{19}$ Ethanol also causes the structural changes in red blood cells because it has direct toxic effects on marrow. A variety of other red cell modifications occurs in relationship with alcohol dependence, particularly in alcoholic cirrhosis. ${ }^{20}$ The liver disease of any cause results in the elevation of MCV levels, because there is increased destruction of red cells and abnormal synthesis in the congested spleen. ${ }^{21}$ Either haemolysis or bleeding results in increased numbers of young red cells with larger cell volume. MCV measurement is widely available, and $\mathrm{MCV}$ is generally measured routinely wherever a full blood count is requested. Automated cell counts are more accurate than manual methods.

The life-span of a red blood cell is 120 days, it might take several weeks/months for changes in drinking to be reflected in MCV levels. ${ }^{22}$ Chronic excessive drinking alcohol results in the elevation of MCV levels in the absence of liver disease, folate deficiency or blood loss. As of now there are no experimental studies proving that an increase in MCV levels after administration of alcohol in healthy controls.

Meerkerk et al revealed that there is no increase in MCV levels in irregular excessive drinkers. ${ }^{23}$ The MCV levels may continue to upswing upon cessation of drinking alcohol in alcohol dependence. ${ }^{24}$ This is because there is increased numbers of reticulocytes due to the marrow begins to recover..$^{25}$

\section{Conclusion}

Our study showed that there is rise in levels of mean corpuscular volume and gamma glutamyl transferase in Alcoholic liver diseases. The MCV $\geq 100$ $\mathrm{fl} / \mathrm{L}$ and GGT $\geq 25 \mathrm{IU} / \mathrm{L}$ show a strong bond with ALD. The results of our study also showed that the ratio AST: ALT > 2 is strongly associated with alcoholic liver diseases along with significant rise in both parameters. Both GGT and MCV can be used for additional diagnostic markers for the diagnosis of liver disease caused by alcoholism. There is still a need for continuous high quality research to maximize the value that can be obtained from traditional markers and to cope with the demographic changes and contextual comorbidity of our population.

\section{References}

1. Lieber CS. Medical disorders of alcoholism. New Engl J Med. 1995:333:1058-1065.

2. Ryback SR, Eckardt JM, Felsher B, Raw RR. Biochemical and hematologic correlates of alcoholism and liver disease. JAMA. 1982;248:2261-5.

3. Robert O'Shea, Dasarathy MC, Cullough AJ, Alcoholic liver disease. AASLD Practice guidelines. 2010;51(1):307-328.

4. Fauci AS, Braunwald E, Kasper LD, Hauser SL, Longo LD, Jameson JL. Harrisons Principles of Internal Medicine: Alcoholic liver disease, 17th edition United States of America: The McGraw Hill Company. 2008:1969-1972.

5. Rodriguez-Roisin R, Krowka MJ, Herve P, Fallon MB, Pulmonary-hepatic vascular disorders (PHD). Eur Respir. 2004;24(5):861-880.

6. Matoff O, Selinger M, Kaplan M. Hepatic transaminase activity in alcoholic liver disease. GE: 1979;76:1195.

7. Daeppen JB, Schoenfeld - Smith K\& TL, Schuckit MT. Characterization of alcohol dependent subjects with very elevated levels of GGT. J Stud Alcohol. 1999;60:589-94.

8. Adelstein A, White G. Alcoholism and mortality. Population Tendencies, 1976;6:7-13.

9. Rosalki, S. B., Rau, D., Lehmann, D. \& Prentice, M. Determination of serum gamma-glutamyl transpeptidase activity and its clinical applications. Annuals of Clinical Biochemistry. 1970;7:143-147.

10. Whitfield, J. B. Gamma glutamyl transferase. Critical Reviews in Clinical Laboratory Sciences, 2001;38:262355.

11. Daeppen JB, Schoenfeld - Smith K, Smith TL, Schuckit MT. Characterization of alcohol dependent subjects with very elevated levels of GGT. J Stud Alcohol. 1999;60:589-94.

12. Cushman P, Jacob G, Barboriak JJ, Anderson AJ. Biochemical marker for alcoholism: sensitivity problems. Alcohol Clin Exp Res. 1984;8:253-57.

13. Sharpe PC, McBridge R, Archbold GP. Biochemical markers of alcohol abuse. QJM. 1996;89:13744.

14. Clerment RJ, Chalmers TC. The transaminase tests in liver disease. Medicine. 1967;46:197-207.

15. Magarian JG, Lucas ML, Kumar KL. Clinical significance in alcoholic patients of commonly encountered laboratory test results. West J Med. 1992;156:287-94.

16. Cohen JA, Kaplan MM. The SGOT/ SGPT ratio in liver disease. Gastroenterology. 1975;69:613.

17. Levin DM, Baker AL, Riddel RH. Non-alcoholic liver disease: overlooked causes of liver injury in patients with heavy alcohol consumption. Am J Med. 1979;66:129-34.

18. Pasqualetti P, Festuccia V, Maccarone C, Di Lauro G, Casale R. Diagnostic value of g-GGT and MCV in chronic hepatitis of alcoholic etiology. Minerva Med: 1995;86;395-402.

19. Wu, A., Chanarin, I. \& Levi, A. J. Macrocytosis of chronic alcoholism. Lancet. $i$ 1974;829-830.

20. Maruyama S, Hirayama C, Yamamoto S, Koda M, Udagawa A, Kadowaki Y, Inoue M, Sagayama A. \& Umeki K. Red blood cell status in alcoholic and nonalcoholic liver disease. Journal of Laboratory and Clinical Medicine. 2001;138:332-337. 
21. Larkin, E. C. \& Watson-Williams, E. J. (1984) Alcohol and the blood. Medical Clinics of North America, 68:105-120.

22. Hasselblatt M., Martin F., Maul O., Ehrenreich H. \& Kernbach-Wighton G. Persistent macrocytosis following abstinence from chronic alcohol use. Journal of the American Medical Association. 2001;286:2946.

23. Meerkerk G. J., Njoo K.H., Bongers I. M., Trienekens P. \& van Oers J. A. Comparing the diagnostic accuracy of carbohydrate-deficient transferrin, gammaglutamyltransferase, and mean cell Volume in a general practice population. Alcoholism, Clinical and Experimental Research. 1999;23:1052-1059.

24. Monteiro M. G. \& Masur J. Monitoring alcoholism treatment: the appropriateness of choice between gamma
GT or MCV evaluation after a short time of abstinence. Alcohol, 1986;3:223-226.

25. Larkin, E. C. \& Watson-Williams, E. J. Alcohol and the blood. Medical Clinics of North America, 1984;68:105120.

How to cite this article: Hemantha K.D.S, Muralidhara K.C.S, Vishwanath H.L. Usefulness of gamma-glutamyl transpeptidase and mean corpuscular volume in alcoholic liver disease. Int J Clin Biochem Res. 2018;5(4):638-641. 